\title{
Functions preserving nonnegativity of matrices
}

\author{
Gautam Bharali \\ Department of Mathematics \\ Indian Institute of Science \\ Bangalore 560012 India
}

\author{
Olga Holtz \\ Department of Mathematics \\ University of California \\ Berkeley, CA 94720 USA
}

November 12, 2005

Key words. Nonnegative inverse eigenvalue problem, circulant matrices, (block) upper-triangular matrices, symmetric matrices, positive definite matrices, entire functions, divided differences.

AMS subject classification. 15A29, 15A48, 15A42

\begin{abstract}
The main goal of this work is to determine which entire functions preserve nonnegativity of matrices of a fixed order $n-$ i.e., to characterize entire functions $f$ with the property that $f(A)$ is entrywise nonnegative for every entrywise nonnegative matrix $A$ of size $n \times n$. Towards this goal, we present a complete characterization of functions preserving nonnegativity of (block) uppertriangular matrices and those preserving nonnegativity of circulant matrices. We also derive necessary conditions and sufficient conditions for entire functions that preserve nonnegativity of symmetric matrices. We also show that some of these latter conditions characterize the even or odd functions that preserve nonnegativity of symmetric matrices.
\end{abstract}

\section{Motivation}

The long-standing inverse eigenvalue problem for nonnegative matrices is the problem of determining, given an $n$-tuple (multiset) $\Lambda$ of complex numbers, whether there exists an entrywise nonnegative matrix $A$ whose spectrum $\sigma(A)$ is $\Lambda$. The literature on the subject is vast and we make no attempt to review it. The interested reader is referred to books [23] and [1], expository papers [3], [9], [18], [19] and references therein, as well as to some very recent papers [4], [20], [27], $[28]$.

The necessary conditions for a given $n$-tuple to be realizable as the spectrum of a nonnegative matrix known so far for arbitrary values of $n$ can be divided into three groups: conditions for nonnegativity of moments, Johnson-Loewy-London inequalities, and Newton's inequalities.

Given an $n$-tuple $\Lambda$, its moments are defined as follows:

$$
s_{m}(\Lambda):=\sum_{\lambda \in \Lambda} \lambda^{m}, \quad m \in \mathbb{N} .
$$

If $\Lambda=\sigma(A)$ for some nonnegative matrix $A$, then $s_{m}(\Lambda)$ is nothing but the trace $\operatorname{tr}\left(A^{m}\right)$, and therefore must be nonnegative. Another basic condition follows from the Perron-Frobenius theory [24], [11]: the largest absolute value $\max _{\lambda \in \Lambda}|\lambda|$ must be the Perron eigenvalue of a realizing 
matrix $A$ and therefore must itself be in $\Lambda$. Finally, the multiset $\Lambda$ must be closed under complex conjugation, being the spectrum of a real matrix $A$. Interestingly, the last two conditions are in fact not independent conditions, but follow from the nonnegativity of moments, as was shown by Friedland in [10]. Thus, there turns out to be just one set of basic conditions

$$
s_{m}(\Lambda) \geq 0 \quad \text { for } m \in \mathbb{N} .
$$

The next set of necessary conditions was discovered independently by Loewy and London in [21] and by Johnson in [17]. These conditions relate moments among themselves as follows:

$$
s_{k}^{m}(\Lambda) \leq n^{m-1} s_{k m}(\Lambda), \quad k, m \in \mathbb{N} .
$$

Newton's inequalities were conjectured in [14] and proved for $M$-matrices in [13]. An $M$-matrix is a matrix of the form $r I-A$, where $A$ is a nonnegative matrix, $r \geq \varrho(A)$, and where $\varrho(A)$ is the spectral radius of $A$ :

$$
\varrho(A):=\max _{\lambda \in \sigma(A)}|\lambda| .
$$

If $M$ is an $M$-matrix of order $n$, then the normalized coefficients $c_{j}(M)$ of its characteristic polynomial defined by

$$
\operatorname{det}(\lambda I-M)=: \sum_{j=0}^{n}(-1)^{j}\left(\begin{array}{l}
n \\
j
\end{array}\right) c_{j}(M) \lambda^{n-j}
$$

must satisfy Newton's inequalities

$$
c_{j}^{2}(M) \geq c_{j-1}(M) c_{j+1}(M), \quad j=1, \ldots, n-1 .
$$

Since the coefficients $c_{j}(M)$ are determined entirely by the spectrum of $M$, and the latter is obtained from the spectrum of a nonnegative matrix $A$ by an appropriate shift, Newton's inequalities form yet another set of conditions necessary for an $n$-tuple to be realizable as the spectrum of a nonnegative matrix. The above three sets of conditions - i.e., nonnegativity of moments, Johnson-LoewyLondon inequalities and Newton's inequalities are all independent of each other but are not sufficient for realizability of a given $n$-tuple (see [13]).

Quite a few sufficient conditions are also known (see, e.g., [33], [19], [10], [3]) as well as certain techniques for perturbing or combining realizable $n$-tuples into new realizable $n$ - or $m$-tuples (where $m \geq n$ ) (see, e.g., [30], [29], [27]). Also, necessary and sufficient conditions on an $n$-tuple to serve as the nonzero part of the spectrum of some nonnegative matrix are due to Boyle and Handelman [2].

Finally, it follows from the Tarski-Seidenberg theorem $[34,26]$ that all realizable $n$-tuples form a semialgebraic set (see also [16]), i.e., for any given $n$, there exist only finitely many polynomial inequalities that are necessary and sufficient for an $n$-tuple $\Lambda$ to be realizable as the spectrum of some nonnegative matrix $A$ (this observation was communicated to us by S. Friedland):

Indeed, each realizable $n$-tuple $\Lambda=\left(\lambda_{1}, \ldots, \lambda_{n}\right)$ is characterized by the condition

$$
\exists A \geq 0 \quad: \quad \operatorname{det}(\lambda I-A)=\prod_{j=1}^{n}\left(\lambda-\lambda_{j}\right) .
$$

The last condition is equivalent to each elementary symmetric function $\sigma_{j}(\Lambda)$ being equal to the $j$ th coefficient of the characteristic polynomial of $A$ multiplied by $(-1)^{j}$ - i.e., to the sum of all 
principal minors of $A$ of order $j$, for $j=1, \ldots, n$. Since the set of all nonnegative matrices is a semialgebraic set in $n^{2}$ entries of the matrix and since each sum of all principal minors of $A$ of order $j$ is a polynomial in the entries of $A$, the lists of coefficients of characteristic polynomials of nonnegative matrices form a semialgebraic set, and hence the $n$-tuples whose elementary symmetric functions match one of those lists also form a semialgebraic set by the Tarski-Seidenberg theorem.

However, despite so many insights into the subject and results obtained so far, the nonnegative inverse eigenvalue problem remains open.

In this paper, we pursue the following idea, which was first expressed by Loewy and London in [21]: Suppose a primary matrix function $f$ is known to map nonnegative matrices of some fixed order $n$ into themselves. Thus $f(A)$ is nonnegative whenever $A$ is. Since $f(\sigma(A))=\sigma(f(A))$, both the spectrum $\sigma(A)$ and its image under the map $f$ must then satisfy all known necessary conditions for realizability - i.e., nonnegativity of moments, the Johnson-Loewy-London inequalities and the Newton inequalities. This enlarges the class of necessary conditions for the nonnegative inverse eigenvalue problem. Describing this larger class would require knowing exactly what functions $f$ preserve nonnegativity of matrices (of a fixed order). That is why we are looking for a complete characterization of such functions. We also believe that this characterization problem can be of independent interest as well.

\section{Outline}

This paper is organized as follows. We make several preliminary observations in Section 4 . In section 5, we characterize the class of functions preserving nonnegativity of triangular and block triangular matrices. It turns out that these are characterized by nonnegativity conditions on their divided differences over the nonnegative reals. In Section 6 , we obtain a characterization of functions preserving nonnegativity of circulant matrices. This characterization is quite different from that in Section 5 - it involves linear combinations of function values taken at certain non-real points of $\mathbb{C}$. In Section 7, we obtain a complete characterization of the class $\mathcal{F}_{n}$ for small values of $n$. The rest of the paper is essentially devoted to functions that preserve nonnegativity of symmetric matrices. After reviewing existing results in that direction in Section 8.1, we obtain new necessary conditions and sufficient conditions in Sections 8.2 and 8.3. Because of a gap between the necessary and the sufficient conditions, which we also point out in Section 8 , these results do not provide a complete characterization of functions preserving nonnegativity of symmetric matrices. We end the paper with a list of several open problems in Section 9, and suggest various approaches to their solution that we have not explored in this paper.

\section{Notation}

We use standard notation $\mathbb{R}^{m \times n}$ for real matrices of size $m \times n, \mathbb{R}_{+}$for nonnegative reals and $\mathbb{Z}_{+}$for nonnegative integers, $A \geq 0(A>0)$ to denote that a matrix $A$ is entrywise nonnegative (positive), and $\sigma(A)$ to denote the spectrum of $A$. For $x \in \mathbb{R}$, we use $\lfloor x\rfloor$ to denote the greatest integer that is less than or equal to $x$. 


\section{Preliminaries}

The main goal of the paper is to characterize functions $f$ such that the matrix $f(A)$ is (entrywise) nonnegative for any nonnegative matrix $A$ of order $n$. Since the primary matrix function $f(A)$ is defined in accordance with values of $f$ and its derivatives on the spectrum of $A$ (see, e.g., [15, Sections $6.1,6.2]$ ), we want to avoid functions that are not differentiable at some points in $\mathbb{C}$. Therefore we restrict ourselves to functions that are analytic everywhere in $\mathbb{C}$, i.e., to entire functions. Thus we consider the class

$$
\mathcal{F}_{n}:=\left\{f \text { entire }: A \in \mathbb{R}^{n \times n}, A \geq 0 \Longrightarrow f(A) \geq 0\right\}
$$

Note right away that the classes $\mathcal{F}_{n}$ are ordered by inclusion:

Lemma 1 For any $n \in \mathbb{N}, \mathcal{F}_{n} \supseteq \mathcal{F}_{n+1}$.

Proof. Let $A$ be a nonnegative matrix of order $n$ and let $f \in \mathcal{F}_{n+1}$. Consider the blockdiagonal matrix $B:=\operatorname{diag}(A, 0)$ obtained by adding an extra zero row and column to $A$. Since $f(B)=\operatorname{diag}(f(A), 0)$, the matrix $f(A)$ must be nonnegative. Thus $f \in \mathcal{F}_{n}$.

Recall that any entire function can be expanded into its Taylor series around any point in $\mathbb{C}$, and that the resulting series converges everywhere (see, e.g., [5]). We will mostly focus on Taylor series of functions in $\mathcal{F}_{n}$ centered at the origin. We start with some simple observations regarding a few initial Taylor coefficients of such a function.

Proposition 2 Let $f(z)=\sum_{j=0}^{\infty} a_{j} z^{j}$ be a function in $\mathcal{F}_{n}$. Then, $a_{j} \geq 0$ for $j=0, \ldots, n-1$.

Proof. For $n=1$, the statement follows from evaluating $f$ at 0 . We now proceed by induction on $n$. If $n>1$ and $f \in \mathcal{F}_{n}$, then by Lemma $1, f \in \mathcal{F}_{n-1}$, whence the inductive hypothesis yields $a_{j} \geq 0$ for $j=0, \ldots, n-2$. Now take

$$
A:=\left[\begin{array}{cccccc}
0 & 1 & 0 & \cdots & 0 & 0 \\
0 & 0 & 1 & \cdots & 0 & 0 \\
0 & 0 & 0 & \cdots & 0 & 0 \\
\vdots & \vdots & \vdots & \ddots & \vdots & \vdots \\
0 & 0 & 0 & \cdots & 0 & 1 \\
0 & 0 & 0 & \cdots & 0 & 0
\end{array}\right] .
$$

Then, the $(1, n)$ entry of the matrix $f(A)$ is equal to $a_{n-1}$, hence must be nonnegative. This finishes the proof.

Corollary 3 A function $f$ is in $\mathcal{F}_{n}$ for all $n \in \mathbb{N}$ if and only if it has the form $f(z)=\sum_{j=0}^{\infty} a_{j} z^{j}$ with $a_{j} \geq 0$ for all $j \in \mathbb{Z}_{+}$.

Proof. One direction follows from Proposition 2. The other direction is trivial: if all terms in the Taylor expansion of $f$ around the origin are nonnegative, then $f(A)$ combines powers of a nonnegative matrix $A$ using nonnegative coefficients, so the resulting matrix is nonnegative.

We now analyze three superclasses of our class $\mathcal{F}_{n}$ :

- entire functions preserving nonnegativity of upper-triangular matrices;

- entire functions preserving nonnegativity of circulant matrices;

- entire functions preserving nonnegativity of symmetric matrices. 


\section{Preserving nonnegativity of (block-)triangular matrices}

We first discuss functions preserving nonnegativity of upper- (or lower-)triangular matrices. The characterization that we obtain makes use of the notion of divided differences. The divided difference (see, e.g., [8]) of a function $f$ at points $x_{1}, \ldots, x_{k}$ (which can be thought of as an ordered sequence $\left.x_{1} \leq \cdots \leq x_{k}\right)$ is usually defined via the recurrence relation

$$
f\left[x_{1}, \ldots, x_{k}\right]:= \begin{cases}\frac{f\left[x_{2}, \ldots, x_{k}\right]-f\left[x_{1}, \ldots, x_{k-1}\right]}{x_{k}-x_{1}} & x_{l} \neq x_{k}, \\ f^{(k-1)}\left(x_{1}\right) /(k-1) ! & x_{1}=x_{k} .\end{cases}
$$

Theorem 4 An entire function $f$ preserves nonnegativity of upper-triangular matrices of order $n$ if and only if its divided differences of order up to $n$ are nonnegative over $\mathbb{R}_{+}$, i.e.,

$$
f\left[x_{1}, \ldots, x_{k}\right] \geq 0 \quad \text { for } x_{1}, \ldots, x_{k} \geq 0, \quad k=1, \ldots, n,
$$

or, equivalently, that all derivatives of $f$ of order up to $n-1$ are nonnegative on $\mathbb{R}_{+}$.

Proof. Sufficiency: Let $A=:\left(a_{i j}\right)$ be a nonnegative upper-triangular matrix. Suppose a function $f$ satisfies (1). By [25], [31] (see also [32]), the elements of the matrix $f(A)$ can be written explicitly as

$$
f(A)_{i j}= \begin{cases}f\left(a_{i i}\right) & i=j, \\ \sum_{i<i_{1}<\cdots<i_{k}<j} a_{i i_{1}} \cdots a_{i_{k} j} f\left[a_{i i}, a_{i_{1} i_{1}}, \ldots, a_{i_{k} i_{k}}, a_{j j}\right] & i<j, \\ 0 & i>j .\end{cases}
$$

The divided differences appearing in the sum on the right-hand side are of order not exceeding $n$; hence all the summands, and therefore the sums, are nonnegative.

Necessity: We procced by induction on $n$. If $f$ preserves nonnegativity of upper-triangular matrices of order $n$, it does so also for matrices of order $n-1$. Thus, by our inductive hypothesis, (1) holds up to order $n-1$. To see that all divided differences of order $n$ are also nonnegative over nonnegative reals, consider the matrix $A$ whose first upper diagonal consists of ones, main diagonal of $n$ arbitrary nonnegative numbers $x_{1}, \ldots, x_{n}$, and all of whose other entries are zero. Then, (2) shows that $f(A)_{1 n}=f\left[x_{1}, \ldots, x_{n}\right]$ and must be nonnegative.

Finally, since all divided differences of a fixed order $k$ at points in a domain $D$ are nonnegative if and only if $f^{(k-1)}(x)$ is nonnegative for every point $x \in D$ [8], we see that condition (1) is equivalent to all derivatives of $f$ of order up to $n-1$ being nonnegative on $\mathbb{R}_{+}$. This finishes the proof.

The proofs of (2) in [31] and [25] are based on the following observation.

Result 5 ([31], [25]) A block-triangular matrix of the form

$$
M=\left[\begin{array}{cc}
A & B \\
0 & a
\end{array}\right], \quad a \in \mathbb{C} \backslash \sigma(A),
$$

is mapped to the matrix

$$
f(M)=\left[\begin{array}{cc}
f(A) & (A-a I)^{-1}(f(A)-f(a) I) B \\
0 & f(a)
\end{array}\right]
$$

by a function $f$. 
One can prove an analogous statement in the block-triangular case:

Proposition 6 Let $f$ be an entire function and let

$$
M=\left[\begin{array}{cc}
A & B \\
0 & C
\end{array}\right], \quad \sigma(A) \cap \sigma(C)=\emptyset .
$$

Then,

$$
f(M)=\left[\begin{array}{cc}
f(A) & f(A) X-X f(C) \\
0 & f(C)
\end{array}\right]
$$

where $X$ is the (unique) solution to the equation

$$
A X-X C=B .
$$

Proof. Let $X$ be a solution of the Sylvester equation $A X-X C=B$. Since the spectra of $A$ and $C$ are disjoint, this solution is unique [15, Section 4.4]. Then, $M=T^{-1} \operatorname{diag}(A, C) T$ where

$$
T=\left[\begin{array}{cc}
I & X \\
0 & I
\end{array}\right]
$$

Hence $f(M)=T^{-1} \operatorname{diag}(f(A), f(C)) T$, which proves the proposition.

As an immediate corollary, we obtain an indirect characterization of functions preserving nonnegativity of block-triangular matrices with two diagonal blocks.

Corollary 7 An entire function $f$ preserves nonnegativity of block upper-triangular matrices of the form

$$
\left[\begin{array}{cc}
A & B \\
0 & C
\end{array}\right], \quad A \in \mathbb{R}^{n_{1} \times n_{1}}, \quad C \in \mathbb{R}^{n_{2} \times n_{2}}
$$

if and only if

a) $f \in \mathcal{F}_{N}$, where $N:=\max \left\{n_{1}, n_{2}\right\}$; and

b) $f(A) X-X f(C) \geq 0$ for every $A \in \mathbb{R}^{n_{1} \times n_{1}}, B \in \mathbb{R}^{n_{1} \times n_{2}}, C \in \mathbb{R}^{n_{2} \times n_{2}}$ such that $A, B, C \geq 0$ and satisfy $\sigma(A) \cap \sigma(C)=\emptyset$.

The matrix $X$ in (b) is the (unique) solution to the equation $A X-X C=B$.

Proof. For $f$ to preserve nonnegativity of blocks $A$ and $C$, it has to belong to $\mathcal{F}_{N}$ (keeping in mind Lemma 1). The remainder of our assertion follows from Proposition 6 and the fact that the matrices with nonnegative blocks $A, B, C$, such that the spectra of $A$ and $C$ are disjoint, are dense in the set of all block upper-triangular matrices.

The above proposition, however, does not allow for an explicit formula of the type (1) as in Theorem 4 .

Remark. Note that the results of this section characterize functions preserving nonnegativity of the (block) lower-triangular matrices as well. 


\section{Preserving nonnegativity of circulant matrices}

A circulant matrix (see, e.g., [7]) $A$ is determined by its first row $\left(a_{0}, \ldots, a_{n-1}\right)$ as follows:

$$
\left[\begin{array}{ccccc}
a_{0} & a_{1} & a_{2} & \cdots & a_{n-1} \\
a_{n-1} & a_{0} & a_{1} & \cdots & a_{n-2} \\
a_{n-2} & a_{n-1} & a_{0} & \cdots & a_{n-3} \\
\vdots & \vdots & \vdots & \ddots & \vdots \\
a_{1} & a_{2} & a_{3} & \cdots & a_{0}
\end{array}\right] .
$$

All circulant matrices of size $n$ are polynomials in the basic circulant matrix

$$
\left[\begin{array}{ccccc}
0 & 1 & 0 & \cdots & 0 \\
0 & 0 & 1 & \cdots & 0 \\
0 & 0 & 0 & \cdots & 0 \\
\vdots & \vdots & \vdots & \ddots & \vdots \\
1 & 0 & 0 & \cdots & 0
\end{array}\right]
$$

which implies in particular that any function $f(A)$ of a circulant matrix is a circulant matrix as well. Moreover, the eigenvalues of a circulant matrix are determined by its first row (see [7]) by the formula

$$
\left\{\sum_{j=0}^{n-1} \omega^{k j} a_{j}: k=0, \ldots, n-1\right\}, \quad \text { where } \omega:=e^{2 \pi \mathrm{i} / n} .
$$

Hence the eigenvalues of $f(A)$ are

$$
\left\{f\left(\sum_{j=0}^{n-1} \omega^{k j} a_{j}\right): k=0, \ldots, n-1\right\} .
$$

Thus, the elements $\left(f_{0}, \ldots, f_{n-1}\right)$ of the first row of $f(A)$ can be read off from its spectrum:

$$
f_{l}=\frac{1}{n} \sum_{k=0}^{n-1} \omega^{-l k} f\left(\sum_{j=0}^{n-1} \omega^{j k} a_{j}\right), \quad l=0, \ldots, n-1 .
$$

This argument proves the following theorem.

Theorem 8 For an entire function $f$ to preserve nonnegativity of circulant matrices of order $n$, it is necessary and sufficient that

$$
\sum_{k=0}^{n-1} \omega^{-l k} f\left(\sum_{j=0}^{n-1} \omega^{j k} a_{j}\right) \geq 0 \quad \text { whenever } a_{j} \geq 0, j=0, \ldots, n-1, \text { where } \omega=e^{2 \pi \mathrm{i} / n} .
$$

\section{Characterization of $\mathcal{F}_{n}$ for small values of $n$}

We now focus of the function classes $\mathcal{F}_{n}$ for small values of $n$. Recall the inclusion $\mathcal{F}_{n+1} \subseteq \mathcal{F}_{n}$ from Lemma 1, which means that all conditions satisfied by the functions from $\mathcal{F}_{n}$ get inherited by the functions from $\mathcal{F}_{n+1}$. Thus we need to find out precisely how to strengthen the conditions that determine $\mathcal{F}_{n}$ to get to the next class $\mathcal{F}_{n+1}$. 


\subsection{The case $n=1$}

A function $f$ is in $\mathcal{F}_{1}$ if and only if $f$ maps nonnegative reals into themselves. While this statement is in a way a characterization in itself, if $f$ is an entire function with finitely many zeros, we can give a description of the form that $f$ takes. For such $f$, the proposition below serves as an alternative characterization.

Proposition 9 A function $f$ having finitely many zeros is in $\mathcal{F}_{1}$ if and only if it has the form

$$
f(z)=g(z) \prod_{\alpha, \beta}\left((z+\alpha)^{2}+\beta^{2}\right) \prod_{\gamma}(z+\gamma),
$$

where the $\alpha$ 's and the $\beta$ 's are arbitrary reals, the $\gamma$ 's are nonnegative, and $g$ is an entire function that has no zeros in $\mathbb{C}$ and is positive on $\mathbb{R}_{+}$.

Proof. First note that since $f$ takes real values over the nonnegative reals, all its zeros occur in conjugate pairs. Moreover, while the multiplicity of the real negative zeros is not resticted in any way, the nonnegative zeros must occur with even multiplicities. This produces exactly the factors recorded in (4), with nonnegative zeros corresponding to $\beta=0$. After factoring out all the linear factors, we are left with an entire function - which we call $g(z)$ - that has no zeros, and takes only positive values on $\mathbb{R}_{+}$. This gives us the expression (4).

Remark. Incidentally, all polynomials $f$ that take only positive values on $\mathbb{R}_{+}$are characterized by a theorem due to Poincaré and Pólya (see, e.g., [6, p.175]): there exists a number $N \in \mathbb{Z}_{+}$such that the polynomial $(1+z)^{N} f(z)$ must have positive coefficients. Since we include non-polynomial functions into our class $\mathcal{F}_{1}$, and since we allow functions to have zeros in $\mathbb{R}_{+}$, the Poincaré-Pólya characterization is not directly relevant to our setup.

\subsection{The case $n=2$}

We just saw that functions in $\mathcal{F}_{1}$ are characterized by one inequality, viz.

$$
f(x) \geq 0 \quad \forall x \geq 0 .
$$

In this subsection we will see that functions in $\mathcal{F}_{2}$ are characterized by two inequalities, one involving a divided difference. We make some preliminary observations first. These observations are valid for all values of $n$.

Lemma 10 An entire function $f$ belongs to $\mathcal{F}_{n}$ if and only if it maps positive matrices of order $n$ into nonnegative matrices.

Proof. This is simply due to the continuity of $f$, since the set of strictly positive matrices is dense in the set of all nonnegative matrices of order $n$.

Lemma 11 For any matrix function $f$, any permutation matrix $P$ and any diagonal matrix $D$ with positive diagonal elements, $f(A)$ is nonnegative if and only if $f\left(P D A(P D)^{-1}\right)$ is nonnegative.

Proof. Note that $(P D) f(A)(P D)^{-1}=f\left(P D A(P D)^{-1}\right)$ and that both matrices $P D$ and $(P D)^{-1}$ are nonnegative. So, $f(A)$ is nonnegative if and only if the matrix $f\left(P D A(P D)^{-1}\right)$ is nonnegative. ( 
Corollary 12 An entire function $f$ belongs to $\mathcal{F}_{2}$ if and only if it maps positive symmetric matrices of order 2 into nonnegative matrices.

Proof. A strictly positive $2 \times 2$ matrix $A$ can be symmetrized by using the transformation $D A D^{-1}$, where $D$ is a diagonal matrix with positive diagonal elements. Thus, Lemmas 10 and 11 imply that $f(A)$ is nonnegative for all strictly positive, and hence for all nonnegative matrices $A$ of order 2 , if and only if $f(A)$ is nonnegative for all symmetric matrices.

Now we are in a position to prove a characterization theorem for the class $\mathcal{F}_{2}$.

Theorem 13 An entire function $f$ is in $\mathcal{F}_{2}$ if and only if it satisfies the conditions

$$
\begin{aligned}
f(x+y)-f(x-y) & \geq 0 \quad \forall x, y \geq 0 \\
(x+y-z) f(x-y)+(z-x+y) f(x+y) & \geq 0 \quad \forall x \geq z \geq 0, y \geq x-z
\end{aligned}
$$

or, equivalently, if $f$ satisfies (6) and the condition

$$
(x+y) f(x-y)+(y-x) f(x+y) \geq 0 \quad \forall y \geq x \geq 0 .
$$

Proof. The condition (6) is one of the two necessary conditions (3) in case $n=2$. Therefore, we need to check that the condition (7) is also necessary and that both together are sufficient. Then we also need to check that conditions (6) and (7) are equivalent to conditions (6) and (8).

By Corollary 12, we can restrict ourselves to the case when $A$ is a positive symmetric matrix, i.e., when

$$
A=\left[\begin{array}{cc}
a_{11} & b \\
b & a_{22}
\end{array}\right], \quad a_{11}, b, a_{22}>0
$$

Since the value of $f$ at $A$ coincides with the value of its interpolating polynomial of degree 1 with nodes of interpolation chosen at the eigenvalues of $A$ [15, Sections 6.1,6.2], we get

$$
f(A)=f\left[r_{1}\right]+f\left[r_{1}, r_{2}\right]\left(A-r_{1} I\right),
$$

where

$$
r_{j}:=\frac{a_{11}+a_{22}}{2}+(-1)^{j} \frac{\sqrt{\left(a_{11}-a_{22}\right)^{2}+4 b^{2}}}{2}, \quad j=1,2 .
$$

So, the off-diagonal entries of $f(A)$ are equal to

$$
f\left[r_{1}, r_{2}\right] b,
$$

while the diagonal entries are

$$
f\left[r_{1}, r_{2}\right]\left(a_{j j}-r_{1}\right)+f\left(r_{1}\right), \quad j=1,2 .
$$

Writing

$$
\begin{aligned}
x & :=\frac{a_{11}+a_{22}}{2} \\
y & :=\frac{\sqrt{\left(a_{11}-a_{22}\right)^{2}+4 b^{2}}}{2} \\
z & :=\min \left(a_{11}, a_{22}\right),
\end{aligned}
$$


we see that the characterization for $\mathcal{F}_{2}$ consists precisely of conditions $(6)$ and $(7)$.

It remains to prove that (6) and (7) are equivalent to (6) and (8). By simply taking $z=0$ in (7), we see that (7) implies (8). So let us now assume (6) and (8). We begin by stating a simple auxiliary fact. Taking $x=0$ and $y>0$ in (6) and (8), we get $f(y) \pm f(-y) \geq 0 \forall y>0$. We conclude from this that $f(y) \geq 0$ whenever $y \geq 0$ - i.e., that $f$ satisfies (5).

First consider $y$ lying in the range $x-z \leq y \leq x$. In this case, we get

$$
\begin{aligned}
& (x+y-z) f(x-y)+(z-x+y) f(x+y) \\
& \quad=(y-(x-z))(f(x+y)-f(x-y))+2 y f(x-y) \geq 0 \quad \text { for } x \geq z \geq 0 .
\end{aligned}
$$

The nonnegativity of the second term above is a consequence of (5), since $x-y$ is nonnegative in this case. Now if $y \geq x$, then (6) and (8) simply imply that

$$
\begin{aligned}
& (x+y-z) f(x-y)+(z-x+y) f(x+y) \\
& =((x+y) f(x-y)+(y-x) f(x+y))+z(f(x+y)-f(x-y)) \geq 0 \\
& \quad \text { for } y \geq x \geq z \geq 0 .
\end{aligned}
$$

The last two inequalities show that (6) and (8) imply (6) and (7). This finishes the proof.

\section{Preserving nonnegative symmetric matrices}

We now focus on the characterization problem for the class of entire functions that preserve nonnegativity of symmetric matrices. We begin by recalling known facts about functions that preserve nonnegative symmetric matrices that are in addition nonnegative definite, i.e., have only nonnegative eigenvalues.

\subsection{Preserving nonnegative definite nonnegative symmetric matrices}

Interestingly, the condition necessary and sufficient for preserving nonnegative symmetric matrices that are nonnegative definite turns out to be exactly the same as the condition for preserving upper(or lower-)triangular nonnegative matrices.

The characterization of functions that preserve the class of nonnegative definite, entrywise nonnegative symmetric matrices is due to Micchelli and Willoughby [22]. We next state a version of their result that is useful for our purposes.

Result 14 (version of [22, Corollary 3.1]) An entire function $f$ preserves the class of nonnegative definite, entrywise nonnegative symmetric matrices if and only if all the divided differences of $f$ of order up to $n$ are nonnegative over $\mathbb{R}_{+}$, i.e., $f$ satisfies (1) or, equivalently, all derivatives $f^{(j)}$ of $f$ up to order $n-1$ are nonnegative on $\mathbb{R}_{+}$.

The proof of Result 14 in [22] relies on two facts. The first is that $f(A)$ coincides with the interpolating polynomial of $f$, with nodes at the eigenvalues of $A$, evaluated at $A$, i.e. that

$$
f(A)=f\left[r_{1}\right] I+f\left[r_{1}, r_{2}\right]\left(A-r_{1} I\right)+\cdots+f\left[r_{1}, \ldots r_{n}\right]\left(A-r_{1} I\right) \cdots\left(A-r_{n-1} A\right) .
$$

The second fact is the entrywise nonnegativity of all matrix products

$$
\left(A-r_{1} I\right) \cdots\left(A-r_{j} I\right), \quad j=1, \ldots n-1,
$$


which holds under the assumption that the eigenvalues $r_{1}, \ldots r_{n}$ of $A$ are ordered

$$
r_{1} \leq r_{2} \leq \cdots \leq r_{n}
$$

Observe, however, that conditions (1) are not sufficient for a function to preserve nonnegativity of all nonnegative symmetric matrices. Indeed, let $n=2$ and let

$$
f(x)=1+x+\frac{1}{2} x^{2}-\frac{2}{3} x^{3}+\frac{1}{4} x^{4} .
$$

This function satisfies the condition (1) with $n=2$, but it maps the matrix

$$
\left[\begin{array}{cc}
0 & M \\
M & 0
\end{array}\right]
$$

which is not nonnegative definite, to a matrix with negative off-diagonal entries when $M>0$ is chosen to be sufficiently large. In fact, any $M>\sqrt{3 / 2}$ will produce a matrix with negative entries.

Motivated by Result 14, we would therefore like to find out what conditions are necessary and sufficient for a function to preserve nonnegativity of a nonnegative symmetric matrices. We begin, in the next subsection, by analyzing even and odd functions.

\subsection{Even and odd functions preserving nonnegativity of symmetric matrices}

Using the Micchelli-Willoughby result - i.e., Result 14 from the previous section - and an auxiliary result from [12], we shall obtain a characterization of even and odd functions that preserve nonnegativity of symmetric matrices. Our proof below will require the notion of a Jacobi matrix and that of a symmetric anti-bidiagonal matrix. A Jacobi matrix is a real, nonnegative definite, tridiagonal symmetric matrix having positive subdiagonal entries. A matrix $A$ is called a symmetric anti-bidiagonal matrix if it has the form

$$
A=\left[\begin{array}{ccccc}
0 & 0 & \cdots & 0 & a_{n} \\
0 & 0 & \cdots & a_{n-2} & a_{n-1} \\
\vdots & \vdots & . & \vdots & \vdots \\
0 & a_{n-2} & \cdots & 0 & 0 \\
a_{n} & a_{n-1} & \cdots & 0 & 0
\end{array}\right], \quad a_{1}, \ldots, a_{n} \in \mathbb{R} .
$$

We make use of the next two results, from [22] and from [12].

Result 15 ([22]) A matrix function $f$ preserves nonnegativity of symmetric nonnegative definite matrices of order $n$ if and only if it maps Jacobi matrices of order $n$ into nonnegative matrices or, equivalently, if the divided differences of $f$ up to order $n$ satisfy (1) for each ordered $n$-tuple $x_{1} \leq x_{2} \leq \cdots \leq x_{n}$ of eigenvalues of a Jacobi matrix.

The above result is not stated in precisely these words in [22], but it is easily inferred - it lies at the heart of the proof of [22, Theorem 2.2]. In addition, we shall also need the following result:

Result 16 (Corollary 3, [12]) Let $\mathcal{M}$ be a positive real $n$-tuple. Then, there exists a Jacobi matrix that realizes $\mathcal{M}$ as its spectrum and has a symmetric anti-bidiagonal square root of the form (10) with all $a_{j}$ 's positive. 
We are now in a position obtain a characterization of even and odd matrix functions that are of interest to us.

Theorem 17 An even entire function $f(z)=: g\left(z^{2}\right)$ preserves nonnegativity of symmetric matrices of order $n$ if and only if the divided differences of $g$ up to order $n$ are nonnegative on $\mathbb{R}_{+}-i$.e., if $g$ satisfies (1). An odd function $f(z)=: z h\left(z^{2}\right)$ preserves nonnegativity of symmetric matrices of order $n$ if and only if $h$ satisfies (1).

Proof. Let $f$ be even. Then, $f(z)=g\left(z^{2}\right)$ for some entire function $g$. If a matrix $A$ is entrywise nonnegative symmetric, then $A^{2}$ is entrywise nonnegative, symmetric, and nonnegative definite. By Result 14, if $g$ satisfies (1), then $g\left(A^{2}\right)$ is nonnegative. To prove the converse, consider an arbitrary $n$-tuple $\mathcal{M}$ of positive numbers. We can think of $\mathcal{M}$ as being ordered

$$
\mathcal{M}=\left(x_{1}, \ldots, x_{n}\right), \quad x_{1} \leq \cdots \leq x_{n} .
$$

By Result 16, there exists a nonnegative symmetric anti-bidiagonal matrix $A$ such that $A^{2}$ is a Jacobi matrix with spectrum $\mathcal{M}$. Then, by Result 15, the divided differences of $g$ must be nonnegative when evaluated at the first $k$ points of $\mathcal{M}$, for each $k=1, \ldots, n$. This implies, by the standard density reasoning, that all divided differences of $g$ must be nonnegative over $\mathbb{R}_{+}$.

Now let $f$ be odd. Then, $f(z)=z h\left(z^{2}\right)$ for some entire function $h$. If all the divided differences of $h$ up to order $n$ are nonnegative, then by the same argument as above, $h\left(A^{2}\right)$ is nonnegative for each symmetric nonnegative matrix $A$, and multiplication of $h\left(A^{2}\right)$ by a nonnegative matrix $A$ produces a nonnegative matrix again. To prove the converse, we use induction and a technique from [22]. Since $f$ has to preserve nonnegativity of symmetric matrices of order $n-1$ as well, we can assume the nonnegativity of the divided differences of orders $k=1, \ldots, n-1$. To prove that the $n$th divided difference is nonnegative, let $\mathcal{M}$ be an arbitrary positive $n$-tuple (11). As above, by Result 16, there exists a symmetric anti-bidiagonal matrix $A$ such that $A^{2}$ is a Jacobi matrix with spectrum $\mathcal{M}$. By [22], formula (9) shows that the $(1, n)$ entry of the function $h\left(A^{2}\right)$ is a positive multiple of $f\left[x_{1}, \ldots, x_{n}\right]$, hence the $(1,1)$ entry of the product $h\left(A^{2}\right) A$ is again a positive multiple of $f\left[x_{1}, \ldots, x_{n}\right]$. Thus the $n$th divided difference has to be nonnegative as well, which finishes the proof.

This theorem provides a rather natural characterization of even and odd functions that preserve nonnegativity of symmetric matrices in terms of their divided differences. However, the "natural" idea, that the even and odd parts of any entire function that preserves nonnegativity of symmetric functions must be also nonnegativity-preserving, turns out to be wrong. Here is an example that illustrates why that may not be the case.

Example 18 Let

$$
f(z):=\alpha+\beta z-z^{3}+z^{5}+\gamma z^{6},
$$

where $\beta>1 / 4$, and $\alpha, \gamma>0$ are chosen to be so large that $f(x) \geq 0$ for all $x \in \mathbb{R}$ and $f^{\prime}(x) \geq 0$ for all $x \in \mathbb{R}_{+}$. Then, $f$ preserves nonnegativity of symmetric matrices of order 2 , but its odd part $f_{\text {odd }}$ does not.

Proof. The function $f$ satisfies conditions (6) and (8). Indeed, since $f \geq 0$ on $\mathbb{R}$, we have

$$
(t+s) f(-t)+t f(t+s) \geq 0 \quad \forall s, t \geq 0,
$$


which is equivalent to condition (8). Now, the odd part of $f$ is given by

$$
f_{\text {odd }}(z)=\beta z-z^{3}+z^{5}=: z h\left(z^{2}\right) .
$$

Since $\beta>1 / 4, h(x)>0$ for all $x \in \mathbb{R}$. Since $f$ is monotone increasing on $\mathbb{R}_{+}$, we have

$$
f(s+t)-f(-s) \geq f(s)-f(-s)=2 f_{\text {odd }}(s) \geq 0 \quad \forall s, t \geq 0,
$$

which yields condition (6). Thus, by Theorem 13, $f$ preserves nonnegativity of symmetric matrices of order 2. However,

$$
h^{\prime}(x)=2 x-1<0 \quad \text { for } x<1 / 2 .
$$

Therefore, by Theorem 17, $f_{\text {odd }}$ does not preserve nonnegativity of symmetric functions of order 2 .

We conclude this section with a simple observation about even and odd parts of a nonnegativitypreserving function.

Proposition 19 If an entire function $f$ preserves nonnegativity of symmetric functions of order $n$, then its even and odd parts $f_{\text {odd }}$ and $f_{\text {even }}$ preserve nonnegativity of matrices of order $\lfloor n / 2\rfloor$.

Proof. For $n$ even, consider matrices of the form

$$
A=\left[\begin{array}{cc}
0 & B \\
B & 0
\end{array}\right]
$$

and for $n$ odd, matrices of the form

$$
A=\operatorname{diag}\left(\left[\begin{array}{cc}
0 & B \\
B & 0
\end{array}\right], 0\right)
$$

where $B$ is an $\lfloor n / 2\rfloor \times\lfloor n / 2\rfloor$ symmetric nonnegative matrix. Since

$$
\begin{gathered}
f(A)=\left[\begin{array}{cc}
f_{\text {even }}(B) & f_{\text {odd }}(B) \\
f_{\text {odd }}(B) & f_{\text {even }}(B)
\end{array}\right] \quad \text { for } n \text { even, } \\
f(A)=\operatorname{diag}\left(\left[\begin{array}{cc}
f_{\text {even }}(B) & f_{\text {odd }}(B) \\
f_{\text {odd }}(B) & f_{\text {even }}(B)
\end{array}\right], 0\right) \quad \text { for } n \text { odd, }
\end{gathered}
$$

the see that $f_{\text {even }}$ and $f_{\text {odd }}$ must preserve nonnegativity of symmetric functions of order $\lfloor n / 2\rfloor$.

\subsection{Other necessary conditions}

Results from [12] allow us to derive an additional set of necessary conditions. The motivation behind these conditions is as follows. We believe that the power of Results 15 and 16 - or rather, the methods behind those results - have not been exhausted by Theorem 17. Our next theorem is presented as an illustration of this viewpoint. On comparison with Theorem 13, we find that the conditions derived in our next theorem constitute a complete characterization for the functions of interest in the $n=2$ case. To derive these new necessary conditions, we will need the following two results. 
Result 20 (Theorem 1, [12]) A real $n$-tuple $\Lambda$ can be realized as the spectrum of a symmetric anti-bidiagonal matrix (10) with all $a_{j}$ 's positive if and only if $\Lambda=\left(\lambda_{1}, \ldots, \lambda_{n}\right)$ satisfies

$$
\lambda_{1}>-\lambda_{2}>\lambda_{3}>\cdots>(-1)^{n-1} \lambda_{n}>0 .
$$

Lemma 21 Let $A$ be a symmetric anti-bidiagonal matrix of order $n$, and let $A_{i j}^{p}$ denote the $(i, j)$ entry of $A^{p}$. Then

a) The $(i, j)$ entry of $A^{2 q-1}$ is zero whenever $2 \leq i+j \leq(n-q+1), q \geq 1$.

b) The $(i, j)$ entry of $A^{2 q}$ is zero whenever $1+q \leq j-i \leq n-1, q \geq 1$.

c) Adopting the notation in (10) for the entries of $A$,

$$
\begin{aligned}
A_{1, n-q+1}^{2 q-1} & =a_{n} a_{n-1} \ldots a_{n-2 q+2}, & & 1 \leq q \leq\lfloor(n+1) / 2\rfloor, \\
A_{1,1+q}^{2 q} & =a_{n} a_{n-1} \ldots a_{n-2 q+1}, & & 1 \leq q \leq\lfloor n / 2\rfloor .
\end{aligned}
$$

Proof. We proceed by induction on $q$. Note that (a), (b) and (c) are obvious when $q=1$. Let us now assume that (a) and (b) are true for some $q<n-3$. Note that since $A$ is anti-bidiagonal,

$$
A_{i j}^{2 q+1}=A_{i, n-j+1}^{2 q} A_{n-j+1, j}+A_{i, n-j+2}^{2 q} A_{n-j+2, j} .
$$

However, if $i+j \leq(n-(q+1)+1)$, then

$$
(n-j+2)-i \geq(n-j+1)-i \geq q+1 .
$$

Applying our inductive hypothesis on (b), we conclude from the above inequalities that the righthand side of (14) reduces to zero when $i+j \leq(n-(q+1)+1)$. Thus, (a) is established for $q+1$.

We establish (b) for $q+1$ in a similar fashion. We note that

$$
A_{i j}^{2 q+2}=A_{i, n-j+1}^{2 q+1} A_{n-j+1, j}+A_{i, n-j+2}^{2 q+1} A_{n-j+2, j} .
$$

When $j-i \geq 1+(q+1)$, then

$$
i+(n-j+1) \leq i+(n-j+2) \leq n-(q+1)+1 .
$$

Since we just established (a) for $q+1$, the above inequalities tell us that the right-hand side of (15) reduces to zero when $j-i \geq 1+(q+1)$. Thus, (b) too is established for $q+1$. By induction, (a) and (b) are true for all relevant $q$.

Part (c) now follows easily by substituting $i=1$ and $j=n-q$ into equation (14) to carry out the inductive step for (12), and by substituting $i=1$ and $j=q+2$ into equation (15) to carry out the inductive step for (13).

We can now present the aforementioned necessary conditions.

Theorem 22 If an entire function $f$ preserves nonnegativity of symmetric matrices of order $n$, $n \geq 2$, then, for each ordered $n$-tuple $\left(x_{1}, \ldots, x_{n}\right)$ where

$$
x_{1}>-x_{2}>x_{3}>\cdots>(-1)^{n-1} x_{n}>0,
$$


$f$ must satisfy

$$
f\left[x_{1}, \ldots, x_{n}\right] \geq 0
$$

and for each $k=1, \ldots, n, f$ must satisfy

$$
f\left[x_{1}, \ldots, x_{k-1}, x_{k+1}, \ldots, x_{n}\right]-\left(\sum_{j \neq k} x_{j}\right) f\left[x_{1}, \ldots, x_{n}\right] \geq 0 .
$$

Proof. We choose an $n$-tuple $\left(x_{1}, \ldots, x_{n}\right)$ that satisfies (16). By Result 20, there is a symmetric anti-bidiagonal matrix of the form (10), with all $a_{j}$ 's positive, whose spectrum is $\left(x_{1}, \ldots, x_{n}\right)$. Let us express $f(A)$ using the formula (9), with the substitutions $r_{j}=x_{j}, j=1, \ldots, n$. Then, in view of Lemma 21 , the $(1,\lfloor n / 2\rfloor+1)$ entry of $f(A)$ is $a_{n} a_{n-1} \ldots a_{2} f\left[x_{1}, \ldots, x_{n}\right]$. Since $f$ preserves nonnegativity, and all the $a_{j}$ 's are positive, $f\left[x_{1}, \ldots, x_{n}\right]$ has to be nonnegative. This establishes (17).

To demonstrate (18), we look at the entries of $f(A)$ that are adjacent to the $(1,\lfloor n / 2\rfloor+1)$ entry that was considered above. Let us fix a $k=1, \ldots, n$. This time, however, in using formula (9) to express $f(A)$, we make the following substitutions

$$
r_{j}= \begin{cases}x_{j} & \text { if } j<k \\ x_{j+1} & \text { if } k \leq j<n \\ x_{k} & \text { if } j=n\end{cases}
$$

Our analysis splits into two cases.

Case 1. $n$ is odd: In this case, let us look at the $(1,\lfloor n / 2\rfloor+2)$ entry of $f(A)$. By Lemma 21, and the fact that $n$ is odd, the only power of $A$ that contributes to this entry is $A^{n-2}$. Consequently

$$
\begin{aligned}
f(A)_{1,\lfloor n / 2\rfloor+2} & =\left\{f\left[x_{1}, \ldots, x_{k-1}, x_{k+1}, \ldots, x_{n}\right]-\left(\sum_{j \neq k} x_{j}\right) f\left[x_{1}, \ldots, x_{n}\right]\right\} A_{1,\lfloor n / 2\rfloor+2}^{n-2} \\
& =a_{n} a_{n-1} \ldots a_{3}\left\{f\left[x_{1}, \ldots, x_{k-1}, x_{k+1} \ldots, x_{n}\right]-\left(\sum_{j \neq k} x_{j}\right) f\left[x_{1}, \ldots, x_{n}\right]\right\} .
\end{aligned}
$$

Since $f$ preserves nonnegativity, (18) follows from the above equalities.

Case 2. $n$ is even: In this case, we focus on the $(1,\lfloor n / 2\rfloor)$ entry of $f(A)$. We recover (18) by arguing exactly as above.

In either case, (18) is established, which concludes our proof.

We conclude this section by showing that a subset of the necessary conditions derived above are in fact sufficient to characterize those entire functions that preserve nonnegativity of $2 \times 2$ symmetric matrices. Specifically, we show that

$$
\begin{aligned}
f\left[x_{1}, x_{2}\right] & \geq 0 \text { and } \\
f\left(x_{2}\right)-x_{2} f\left[x_{1}, x_{2}\right] & \geq 0 \text { for all } x_{1}>-x_{2}>0
\end{aligned}
$$

imply the conditions (6) and (8). This is achieved simply by taking some $y>x>0$, making the substitutions $x_{1}=y+x$ and $x_{2}=x-y$, and then invoking continuity to obtain (6) and (8) for all $y \geq x \geq 0$. 


\section{Open problems and further ideas}

We conclude this paper by listing some ideas that we did not pursue, which however may lead to further progress.

One can consider matrices that preserve nonnegativity of other classes of structured matrices, such as Toeplitz or Hankel. However, since these classes are not invariant under the action of an arbitrary matrix function, their matrix functions can be quite difficult to analyze. Also, the eigenstructure of some structured matrices is rather involved, which could be an additional obstacle.

Theorem 1.3 of [31] gives an interesting formula for $f(A)$ when $f$ is a polynomial, which therefore must also be true for entire functions. Precisely, if $A$ is a matrix with minimal polynomial $p_{0}$ and $C$ is the companion matrix of $p_{0}$, then

$$
f(A)=\sum_{j=1}^{n} f(C)_{j 1} A^{j-1} .
$$

In particular, $f(A)$ is nonnegative whenever the first column of $f(C)$ is nonnegative. It would be worthwhile to find out what functions have this property.

Note that the set $\mathcal{F}_{n}$ contains positive constants and is closed under addition, multiplication, and composition. We are not aware of any work on systems of entire functions (or even polynomials) that satisfy this property. Perhaps one could describe a minimal set of generators (with respect to these three operations) that generate such a system.

For example, in the case $n=1$, the generators are positive constants, the function $p_{1}(x)=x$ plus all quadrics of the form $(x-a)^{2}, a>0$. Incidentally, the set of polynomials with nonnegative coefficients is generated by positive constants and $p_{1}(x)=x$. We do not have a characterization of generators for $n \geq 2$.

In particular, $\mathcal{F}_{n}$ is a semigroup with respect to any of these operations, so some general results on semigroups may prove to be useful in our setting. Also note that the set of nonnegative matrices of order $n$, on which $\mathcal{F}_{n}$ acts, is also a semigroup (closed under addition and multiplications), which could also be of potential use.

Finally, both $\mathcal{F}_{n}$ and the set of nonnegative matrices of order $n$ are also cones, so the problem might also have a cone theoretic form. If we consider polynomials instead of entire functions, we can further restrict ourselves to polynomials of degree bounded by a fixed positive integer. Then, we will obtain a proper cone, whose extreme directions may be of interest. The general problem then can also be looked upon in an appropriate similar setting.

\section{Acknowledgments}

We are grateful to Raphael Loewy, Michael Neumann and Shmuel Friedland for helpful discussions.

\section{References}

[1] Abraham Berman and Robert J. Plemmons. Nonnegative matrices in the mathematical sciences, volume 9 of Classics in Applied Mathematics. Society for Industrial and Applied Mathematics (SIAM), Philadelphia, PA, 1994. 
[2] Mike Boyle and David Handelman. The spectra of nonnegative matrices via symbolic dynamics. Ann. of Math. (2), 133(2):249-316, 1991.

[3] Moody T. Chu. Inverse eigenvalue problems. SIAM Rev., 40(1):1-39 (electronic), 1998.

[4] Moody T. Chu and Shu-fang Xu. On computing minimal realizable spectral radii of nonnegative matrices. Numer. Linear Algebra Appl., 12(1):77-86, 2005.

[5] John B. Conway. Functions of one complex variable. II, volume 159 of Graduate Texts in Mathematics. Springer-Verlag, New York, 1995.

[6] John P. D'Angelo. Inequalities from complex analysis, volume 28 of Carus Mathematical Monographs. Mathematical Association of America, Washington, DC, 2002.

[7] Philip J. Davis. Circulant matrices. John Wiley \& Sons, New York-Chichester-Brisbane, 1979.

[8] Carl de Boor. Divided differences. Surveys in Approximation Theory, 1:46-69, 2005.

[9] Patricia D. Egleston, Terry D. Lenker, and Sivaram K. Narayan. The nonnegative inverse eigenvalue problem. Linear Algebra Appl., 379:475-490, 2004.

[10] Shmuel Friedland. On an inverse problem for nonnegative and eventually nonnegative matrices. Israel J. Math., 29(1):43-60, 1978.

[11] Georg F. Frobenius. Über matrizen aus nicht negativen Elementen. In Sitzungsberichte der Königlich Preussischen Akademie der Wissenschaften, pages 456-477. Springer, Berlin, 1912.

[12] Olga Holtz. The inverse eigenvalue problem for symmetric anti-bidiagonal matrices. Linear Algebra Appl., 408:268-274, 2005.

[13] Olga Holtz. M-matrices satisfy Newton's inequalities. Proc. Amer. Math. Soc., 133(3):711-717 (electronic), 2005.

[14] Olga Holtz and Hans Schneider. Open problems on GKK $\tau$-matrices. Linear Algebra Appl., $345: 263-267,2002$.

[15] Roger A. Horn and Charles R. Johnson. Topics in matrix analysis. Cambridge University Press, Cambridge, 1994.

[16] Nathan Jacobson. Lectures in abstract algebra. Vol III: Theory of fields and Galois theory. D. Van Nostrand Co., Inc., Princeton, N.J.-Toronto, Ont.-London-New York, 1964.

[17] Charles R. Johnson. Row stochastic matrices similar to doubly stochastic matrices. Linear and Multilinear Algebra, 10(2):113-130, 1981.

[18] Thomas J. Laffey. Inverse eigenvalue problems for matrices. Proc. Roy. Irish Acad. Sect. A, 95(suppl.):81-88, 1995.

[19] Thomas J. Laffey. Realizing matrices in the nonnegative inverse eigenvalue problem. In Matrices and group representations (Coimbra, 1998), volume 19 of Textos Mat. Sér. B, pages 21-32. Univ. Coimbra, Coimbra, 1999. 
[20] António Leal-Duarte and Charles R. Johnson. Resolution of the symmetric nonnegative inverse eigenvalue problem for matrices subordinate to a bipartite graph. Positivity, 8(2):209-213, 2004 .

[21] Raphael Loewy and David London. A note on an inverse problem for nonnegative matrices. Linear and Multilinear Algebra, 6(1):83-90, 1978/79.

[22] Charles A. Micchelli and R. A. Willoughby. On functions which preserve the class of Stieltjes matrices. Linear Algebra Appl., 23:141-156, 1979.

[23] Henryk Minc. Nonnegative matrices. Wiley-Interscience Series in Discrete Mathematics and Optimization. John Wiley \& Sons Inc., New York, 1988.

[24] Oskar Perron. Zur Theorie der Matrices. Math. Ann., 64(2):248-263, 1907.

[25] B. A. Schmitt. Eine explizite Darstellung der Funktion einer Dreiecksmatrix. Z. Angew. Math. Mech., 59(3):T76-T77, 1979.

[26] A. Seidenberg. A new decision method for elementary algebra. Ann. of Math. (2), 60:365-374, 1954.

[27] Helena Šmigoc. Construction of nonnegative matrices and the inverse eigenvalue problem. Linear Multilinear Algebra, 53(2):85-96, 2005.

[28] Ricardo Soto, Alberto Borobia, and Julio Moro. On the comparison of some realizability criteria for the real nonnegative inverse eigenvalue problem. Linear Algebra Appl., 396:223$241,2005$.

[29] Ricardo L. Soto. Existence and construction of nonnegative matrices with prescribed spectrum. Linear Algebra Appl., 369:169-184, 2003.

[30] George W. Soules. Constructing symmetric nonnegative matrices. Linear and Multilinear Algebra, 13(3):241-251, 1983.

[31] James D. Stafney. Functions of a matrix and their norms. Linear Algebra and Appl., 20(1):8794, 1978.

[32] James D. Stafney. Correction to: "Functions of a matrix and their norms" [Linear Algebra Appl. 20 (1978), no. 1, 87-94; MR 57 \#3162]. Linear Algebra Appl., 39:259-260, 1981.

[33] H. R. Suleĭmanova. Stochastic matrices with real characteristic numbers. Doklady Akad. Nauk SSSR (N.S.), 66:343-345, 1949.

[34] Alfred Tarski. A decision method for elementary algebra and geometry. University of California Press, Berkeley and Los Angeles, Calif., 1951. 